\title{
Foreign body airway obstruction, Incidence, survival and first aid treatment by laymen
}

Theo W. Jensen, Mathias G. Holgersen, Stig N. F. Blomberg, Freddy Lippert, Helle C. Christensen Emergency Medical Services Copenhagen, University of Copenhagen, Copenhagen, Denmark.

\section{BACKGROUND:}

Foreign body airway obstruction (FBAO) is often described as an uncommon cause of Out of Hospital Cardiac Arrest (OHCA) accounting for approximately $1.4 \%$ of all OHCA [1,2]. Reported incidents rates of FBAO causing cardiac arrest are unclear, and first aid by layperson are not well described. The purpose of this investigation is to show incidents rates, action taken and outcome from Denmark.

\section{METHODS:}

Data was collected from the verified 2016-2018 Danish OHCA register, and cases with FBAO prior to OHCA was selected via a direct marking by external validation and advanced text search. Patients reported as indisputably deceased (late signs of death) was excluded. Incidence rates per 100.000 citizens, survival rates to hospital and first aid actions by layperson are presented.

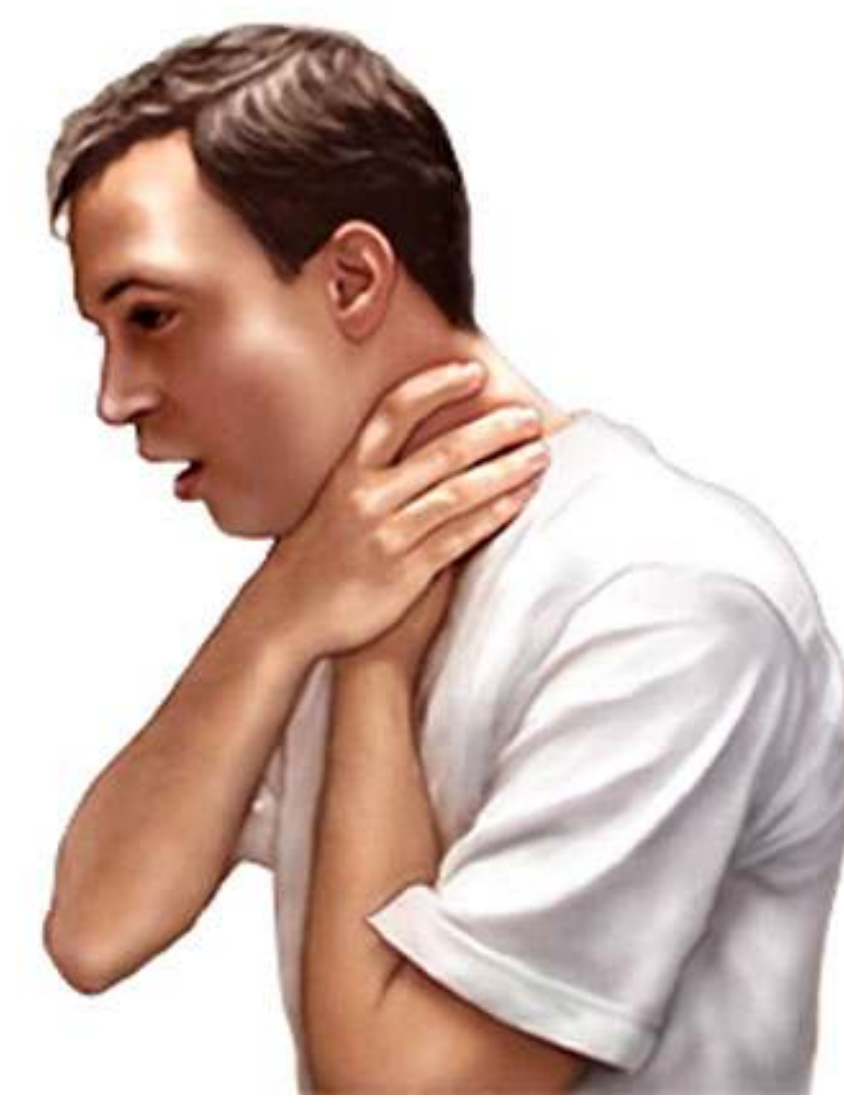

Figur 1.Typical manifestation of patients suffering foreign body airway obstruction.

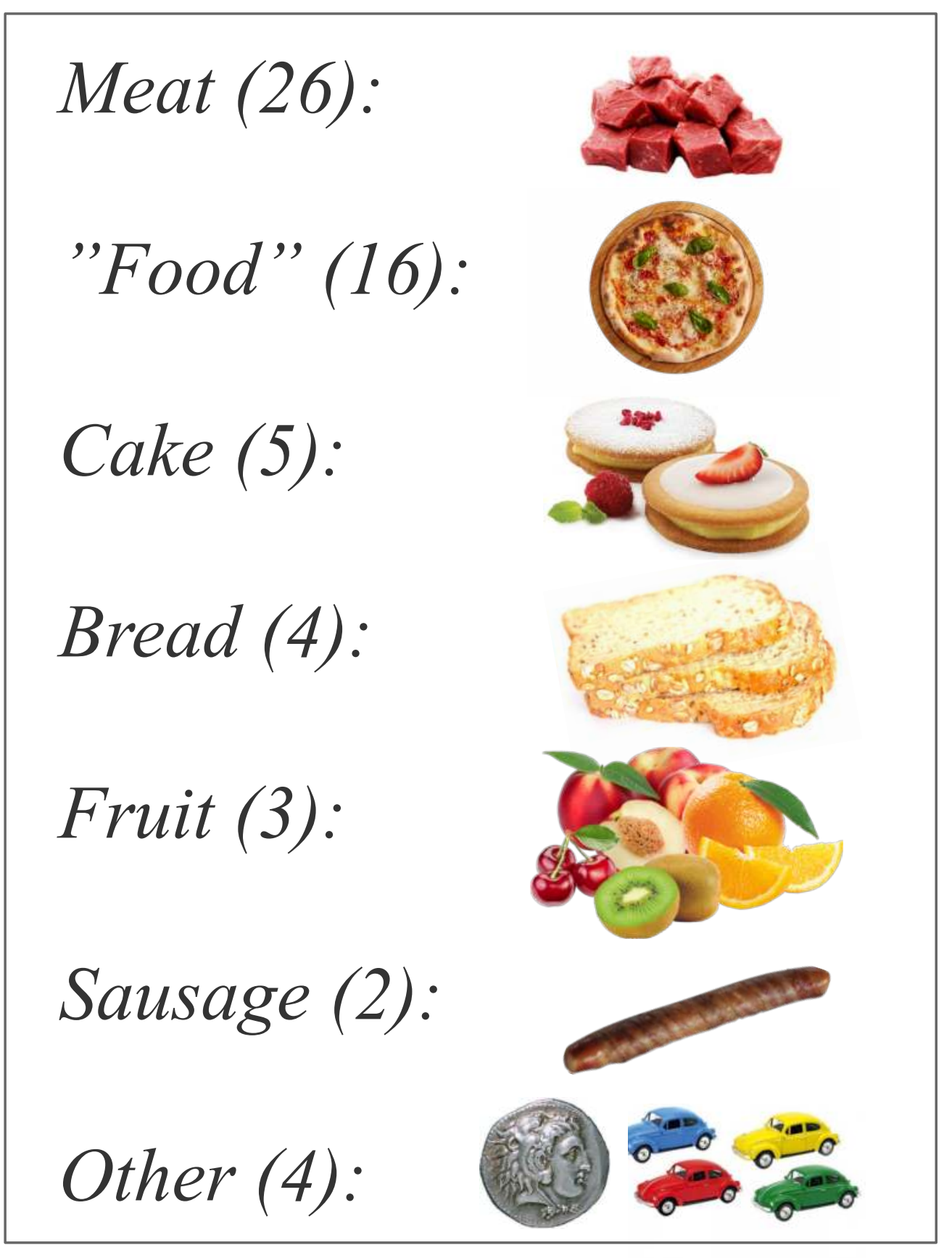

Figur 2. Characteristics of know objects in the presented preliminary sample.

\section{CONCLUSION:}

Data show that FBAO is still an uncommon cause of cardiac arrest in Denmark. There seems to be a positive effect of first aid by laypersons.

\section{RESULTS:}

Copenhagen, Denmark has approximately 1.8 million inhabitants and 4529 indexed and verified OHCA between 2016 and 2018. A search identified $121 \mathrm{OHCA}$ suspected to be caused by FBAO and 63 of these were verified. Incidence rates was 0.9-1.6 per 100.000 inhabitants per year and survival to hospital rate was $65 \%$. Treatment was initiated by laypersons in 43 cases. The most common interventions were CPR and abdominal thrust. All cases involved food items (predominantly meat, $\mathrm{N}: 25)$. Mortality was considerably higher among victims not treated by bystanders $(50 \%$ vs. $30.2 \%)$

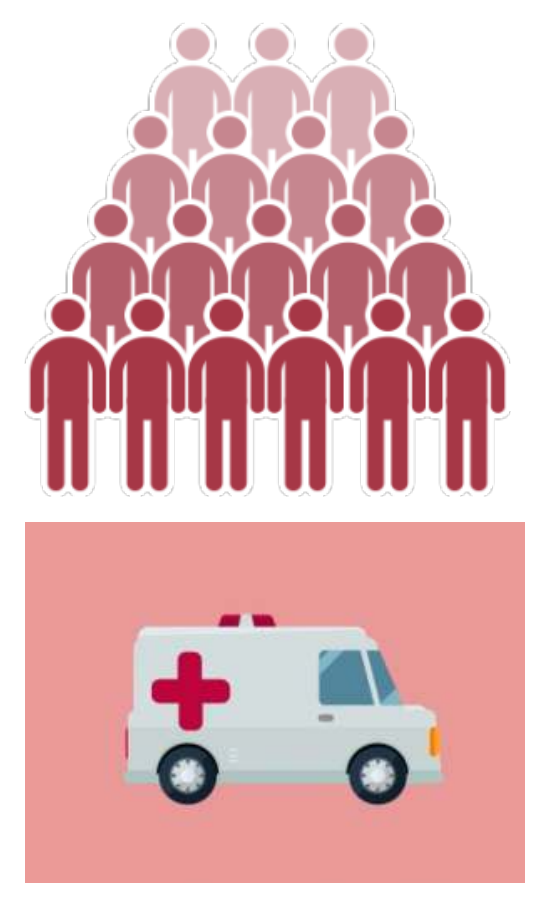

Incidence rate per 100.000 inhabitants

0.9-1.6

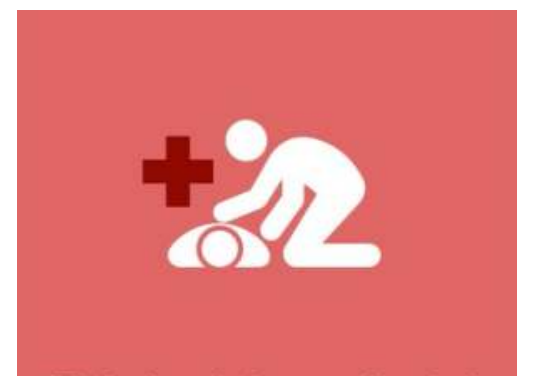

Treated by laypersons

$68 \%$ 